\title{
COMPENSATION OF OSCILLATING INSTANTANEOUS POWER IN MODERN MICROGRIDS BASED ON THE CONSERVATIVE POWER THEORY
}

\author{
José de A. O. Filho ${ }^{1}$, Helmo K. Morale-Paredes ${ }^{1}$, Jakson P. Bonaldo ${ }^{2}$, Augusto M. S. Alonso ${ }^{1,3}$, Fernando \\ P. Marafão ${ }^{1}$, Marcelo G. Simões ${ }^{4}$ \\ ${ }^{1}$ Universidade de São Paulo - USP, Sorocaba - SP, Brazil \\ ${ }^{2}$ Universidade Federal de Mato Grosso - UFMT, Cuiabá - MT, Brazil \\ ${ }^{3}$ Norwegian University of Science and Technology, Trondheim, Norway \\ ${ }^{4}$ Colorado School of Mines, Golden - CO, USA \\ e-mail: jose.olimpio@unesp.br, helmo.paredes@unesp.br, augusto.alonso@unesp.br, jaksonpaulo@ufmt.br, \\ fernando.marafao@unesp.br,msimoes@mines.edu
}

\begin{abstract}
Considering the application of multifunctional grid-tied inverters in modern microgrids, this paper proposes a novel control strategy derived from the Conservative Power Theory (CPT), which makes possible the compensation of instantaneous power oscillations. Such approach is based on the instantaneous power and instantaneous reactive energy terms defined by the CPT, allowing the extraction of oscillating power components directly in the $a b c$ frame. Simulation results are presented to demonstrate the applicability of the control strategy considering the scenario of a weak microgrid with linear, non-linear, and unbalanced loads, as well as comprising a three-phase multi-functional grid-tied inverter with LCL filter. The results show that active power dispatchability can be offered by the inverter, while concomitantly supporting the microgrid to operate at constant instantaneous power. Experimental results comprising a $3.6 \mathrm{kVA}$ inverter prototype also validate the proposed decomposition of oscillating power terms, showing that they can be satisfactorily employed on compensation purposes.
\end{abstract}

$\begin{aligned} & \text { Keywords } \\ & \text { Instantaneous }\end{aligned}$ Power Onservative Power $\begin{array}{r}\text { Theory, } \\ \text { Oscillations, }\end{array}$ Microgrid,
Multifunctional Grid-Tied Inverters, Power Quality.

\section{INTRODUCTION}

The desire for sustainable energy generation has been pushing the proliferation of Renewable Energy Resources (RES) over modern power systems. Moreover, novel regulations and energy policies, such as credit incentives and tax subvention, have supported the spread of RES by means of Distributed Energy Resources (DER) [1]-[3]. As a consequence, the renewable-based electric power generation can now be found under a decentralized approach, occurring under a flexible and controlled manner, by employing power electronic interfaces (PEIs) [4]-[6].

Thus, such PEIs (specially inverters) associated to DER are the main responsible for this new perspective of flexible power conversion in modern electric systems. Additionally, due to the conceptual adoption of multi-functionalities [7],

\footnotetext{
Manuscript received 03/31/2020; first revision 07/08/2020; accepted for publication 08/06/2020, by recommendation of Editor Demercil de Souza Oliveira Jr.. http://dx.doi.org/10.18618/REP.2020.3.0017
}

such electronic equipment is not just able to provide the conversion of active power. Still, they can also offer services for supporting the grid on, for instance, achieving compliance with power quality requirements [8]-[10]. The provision of ancillary services related to the compensation of electrical disturbances, mainly based on multi-functional grid-tied inverters (MFGTI), is most important in scenarios where the grid is weak, consequently being prone to operate under low efficiency or being incapable to provide robust voltage support/stability. The concept of microgrids, although being considered an appealing solution for industrial systems, commercial, rural, and residential area networks [11], can be an example of such a weak power system [12].

A microgrid is comprised of a set of loads and DER elements that may or may not have energy storage, knowing that such items are connected to a distribution grid through a Point of Common Coupling (PCC). Additionally, microgrids can operate in either on-grid or off-grid mode, imposing that its infrastructure should be robust and able to switch between these two operation modes smoothly [13], [14]. Consequently, a grid-interactive PEI [14] endowed with Energy Management System (EMS) [15]-[16] usually interconnects the microgrid with the upstream power system, or voltage-controlled converters comprising energy storage are required to support adequate transient and steady-state operation [17] for both modes. However, for what concerns this paper, only the scenario of a grid-connected microgrid is considered.

Another perspective is one of implementing a microgrid as a low-voltage distribution system [18]. Therefore, such an electric network of limited size must also be able to supply energy to single- and two-phase (linear or non-linear) loads in three-phase systems. For instance, possible loads that may be connected to a three-phase three-wire microgrid are motors, adjustable speed drives, UPSs, beyond other domestic loads that can be connected under phase-to-phase configuration, such as lighting reactors, home appliances, so forth. Although, the majority of such loads likely present a non-linear behavior, draining distorted and unbalanced currents, which may impair voltage quality and deteriorate the operability of other electric equipment.

Also, taking into account that PEIs integrate RES that present intermittent patterns of power generation, the energy generated and consumed may vary significantly through time within a microgrid, creating a dynamic and complex power 
system with a high level of interaction between sources and loads. In this context, the main challenges for operating microgrids are the RES intermittency, as well as the variable load consumption, which consequently causes instantaneous power oscillations [19]-[21]. Such variations can cause braking torque oscillations on rotating machines, trigger tieline power fluctuations [22], also affecting the energy dispatch on wind power generation [23]-[25].

As a consequence, it is of importance to mention that power quality issues in a three-wire three-phase microgrid are associated with harmonics, unbalance, and instantaneous power oscillations. For example, the nature of the instantaneous power and average power of different load configurations, considering the same rated power, is shown in Figure 1. Note that the energy oscillation between the load and the source depends on the load configuration, leading to the urge of developing mitigation strategies that can support the microgrid on achieving a seamless operation for what concerns power oscillations. Therefore, different from classic compensation strategies that aim to mitigate current harmonics and unbalance [10, 26-29], this paper focuses on the instantaneous power oscillation problem to be suppressed by a three-phase MFGTI existing in a grid-connected microgrid.

Thus, this study proposes a novel interpretation of the instantaneous power and reactive energy terms defined by the Conservative Power Theory (CPT) [30], as a new alternative for implementing MFGTIs that can tackle the problem of oscillating instantaneous power. The proposed control algorithm for generating the reference signals for the operation of the MFGTI is obtained directly in $a b c$ frame, without requiring implementation of coordinate transformations or the use of phase-locked-loops (PLL). Therefore, particularly in respect to this latter, the proposed strategy presents inherent advantages such as the provision of a fast dynamic response for the MFGTI control architecture, besides eliminating concerns related to stability matters that are tied to the use of most PLL algorithms [31]. In comparison to [32], the main contribution of this paper lies on bringing a more extensive discussion on the application and capabilities of the proposed control method based on the CPT, also showing additional simulation results, as well as presenting experimental results to validate the feasibility of the strategy.

\section{THE CONSERVATIVE POWER THEORY: BASIC DEFINITIONS}

For the basic definitions of the CPT, let us consider a set of real electrical quantities, which are continuous and periodic (i.e., having period $T$ ), with fundamental frequency $f=1 / T$ and angular frequency $\omega=2 \pi f$. As thoroughly discussed in [30], the CPT proposes an approach in time domain only based on $a b c$ coordinates, defining two main instantaneous power terms. First, the instantaneous power term is described by the scalar product between the voltage $(v)$ and current $(i)$ vectors:

$$
p(t)=\boldsymbol{v} \circ \boldsymbol{i}=\left[\begin{array}{lll}
v_{u} & v_{b} & v_{c}
\end{array}\right] \circ\left[\begin{array}{l}
i_{a} \\
i_{b} \\
i_{c}
\end{array}\right]
$$

Secondly, instantaneous reactive energy is defined as:

$$
w(t)=\widehat{\boldsymbol{v}} \circ \boldsymbol{i}=\left[\begin{array}{lll}
\hat{v}_{u} & \hat{v}_{b} & \hat{v}_{c}
\end{array}\right] \circ\left[\begin{array}{l}
i_{a} \\
i_{b} \\
i_{c}
\end{array}\right]
$$

where, $\widehat{v}$ is a vector that contains the unbiased integrals of phase voltages. This quantity is given by the difference between the time integral and its mean value, as shown in (3).

$$
\hat{v}_{m}=\int_{0}^{t} v_{m}(\tau) d \tau-\frac{1}{T} \int_{0}^{T}\left[\int_{0}^{t} v_{m}(\tau) d \tau\right] d t
$$

The " $m$ " index represents the variables for each phase. Also, according to the CPT, in three-wire three-phase circuits, the phase voltages should be measured using a virtual reference point [33].

The mean values corresponding to (1) and (2) are given respectively by (4) and (5), on which $P$ is the active power and $W$ is the reactive energy and its physical meaning is discussed in [30], [33].

$$
\begin{gathered}
\bar{p}=\frac{1}{T} \int_{0}^{T} p(t) d t=\frac{1}{T} \int_{0}^{T}\left(v_{a} i_{a}+v_{b} i_{b}+v_{c} i_{c}\right) d t=P \\
\bar{w}=\frac{1}{T} \int_{0}^{T} w(t) d t=\frac{1}{T} \int_{0}^{T}\left(\hat{v}_{a} i_{a}+\hat{v}_{b} i_{b}+\hat{v}_{c} i_{c}\right) d t=W
\end{gathered}
$$

Based on the definitions of (4) and (5), the CPT decomposes the load phase currents as the sum of five subcomponents that are: balanced active currents, balanced reactive currents, unbalanced active currents, unbalanced reactive currents, and residual currents [30], [33]. For a sinusoidal voltage operation, independently of balanced or unbalanced characteristics, the active and reactive current components, irrespectively of being balanced or unbalanced related, correspond to a portion of the fundamental current. On the other hand, the remaining part defined by the CPT
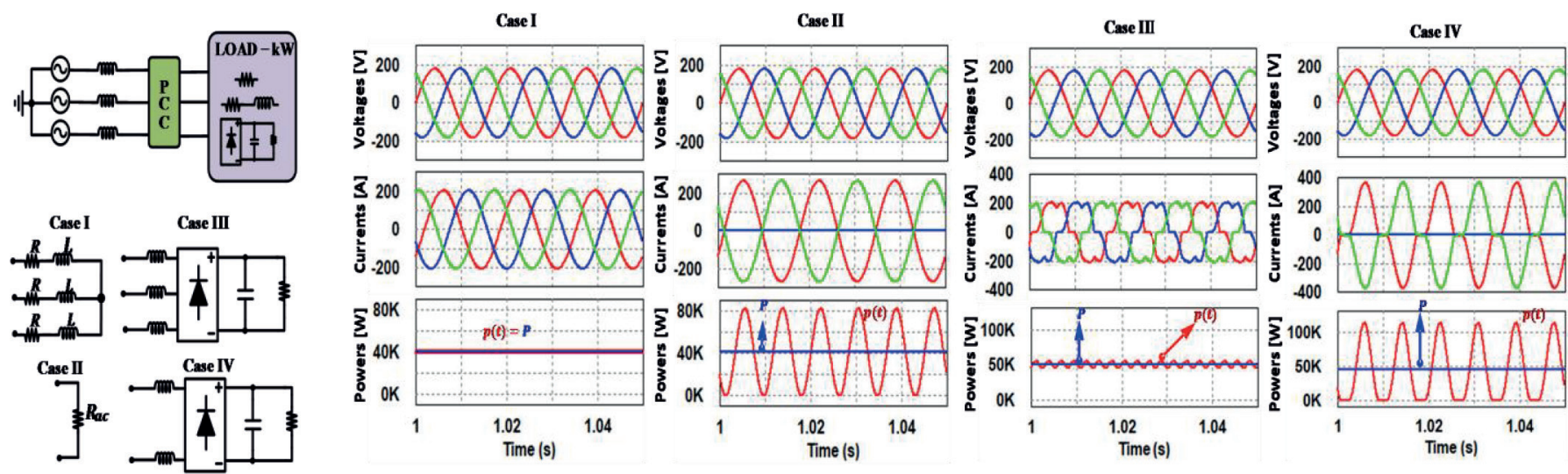

Fig. 1. Time evolution of instantaneous power $p(t)$ and average power $P$ for different load configuration with the same nominal power. 
corresponds to the harmonic components generated by the non-linear loads. These current subcomponents have been satisfactorily applied to make reference signals for singlephase and three-phase active power filters (APF) [34], [35], as well as for MFGTIs [10], [26]. Such decompositions provided by the CPT allow selective and oriented identification of different disturbances on a generic load (non-linearities, unbalances and reactive power [33]). Hence, it is possible to set compensation strategies for undesirable currents with high flexibility level.

In the light of the fact that compensation of oscillating powers in three-wire three-phase systems has not yet been explored in literature based on the CPT, the main contribution of this work is settled. It is important to reinforce that, although other previously proposed strategies provided means to achieve constant instantaneous power in electric circuits [19]-[21], their mathematical and physical interpretations, as well as their implementation for the control of MFGTIs, differ from the CPT's definitions discussed herein. Consequently, the following section aims to analyze the defined power terms within (1), (2), (4) and (5), from the perspective of power oscillations, and their relationship with the generation of control signals for a MFGTI in three-phase three-wire networks.

\section{COMPENSATION STRATEGY AND PROPOSED CONTROL ALGORITHM}

The goal of compensating instantaneous power oscillations by means of the CPT can be devised by the decomposition of (1) and (2), attaining mean and oscillating terms. Therefore, the instantaneous power and reactive energy terms defined in $a b c$ frame result as in (6) and (7), respectively:

$$
\begin{gathered}
p(t)=\bar{p}+\tilde{p} \\
w(t)=\bar{w}+\widetilde{w} .
\end{gathered}
$$

where " $\sim$ " represents the oscillating components of each instantaneous term. The mean components, which are represented by "-_" can be derived by (4) and (5) and are valid independently of voltage and current waveforms. This means that such approach is valid to be applied for both sinusoidal and nonsinusoidal voltage conditions.

The average terms comprised in (3), (6) and (7) can be derived using different structures of digital low-pass filters, similar to traditional methods like the p-q theory [36] or other control strategies for APFs [19]. This choice should always consider the tradeoff between the slow dynamics of infinite impulse response (IIR) low-pass filters and steadystate errors of finite impulse response (FIR) filters (e.g. moving average filters), in case frequency variations are expected and if adaptive structures [37] are not used. However, if well designed, such filters would not impair the performance of the overall control strategy, as it will be further demonstrated.

The instantaneous power, $p(t)$, represents the useful energy per unit of time that flows from the source to the load (or from the load to the source, if negative). Thus, if the mean component of $p(t)$ is positive, it comprises the energy per unit of time that is transferred from the source to the load. On the other hand, the oscillating component $(\tilde{p})$ corresponds to the energy per unit of time that is exchanged between the source and the load. Certainly, the mean value of the oscillating component is zero, but at each instant, it represents a quantity of energy that flows on the electric circuit due to an undesirable current.

Generally, the calculation of the mean active power component $(\bar{p})$ can consider the period of the grid frequency, as in (4). On the other hand, the oscillating component $(\tilde{p})$ corresponds to the components with higher frequencies than that from the grid's fundamental one, or it is given by the components of the negative sequence. Furthermore, also contribute to $\tilde{p}$ the unbalanced loads, non-linear loads and resonances triggered by single- or two-phase converters connected to three-wire three-phase circuits. Particularly, in three-phase electric circuits, with or without neutral wire, where voltages and currents consist only of positive sequence and fundamental components, the energy transfer is unidirectional, usually flowing from source to load. Therefore, the instantaneous power contains just the mean component $(p(t)=\bar{p}=P)$ in such case.

In any other situation, if voltages and currents comprise unbalanced components, the instantaneous power will present mean and oscillating components with a bidirectional flow.

\section{A. Generation of Reference Signals for Compensation of Instantaneous Power Oscillations}

Based on the active power and reactive energy quantities devised from the CPT in (6) and (7), two instantaneous current components, $i_{p}$ and $i_{w}$, can be defined as:

$$
\begin{gathered}
\boldsymbol{i}_{p}=\frac{\bar{p}}{v_{a b c}^{2}}\left[\begin{array}{l}
v_{u} \\
v_{b} \\
v_{c}
\end{array}\right]+\frac{\tilde{p}}{v_{a b c}^{2}}\left[\begin{array}{l}
v_{u} \\
v_{b} \\
v_{c}
\end{array}\right] \\
\boldsymbol{i}_{w}=\frac{\bar{w}}{\hat{v}_{a b c}^{2}}\left[\begin{array}{l}
\hat{v}_{a} \\
\hat{v}_{b} \\
\hat{v}_{c}
\end{array}\right]+\frac{\widetilde{w}}{\hat{v}_{a b c}^{2}}\left[\begin{array}{l}
\hat{v}_{a} \\
\hat{v}_{b} \\
\hat{v}_{c}
\end{array}\right] .
\end{gathered}
$$

where $v_{a b c}^{2}=v_{a}^{2}+v_{b}^{2}+v_{c}^{2}$ and $\hat{v}_{a b c}^{2}=\hat{v}_{a}^{2}+\hat{v}_{b}^{2}+\hat{v}_{c}^{2}$ are the collective instantaneous values of voltage and unbiased integrals of voltage, respectively. Herein, the bold variables represent vector quantities.

Therefore, the current vectors associated with instantaneous terms $[p(t)$ and $w(t)]$ can be decomposed into two subcomponents:

$$
\begin{gathered}
\boldsymbol{i}_{p}=\boldsymbol{i}_{\bar{p}}+\boldsymbol{i}_{\tilde{p}} \\
\boldsymbol{i}_{w}=\boldsymbol{i}_{\bar{w}}+\boldsymbol{i}_{\widetilde{w}} .
\end{gathered}
$$

From a practical understanding, the oscillating components $\left(\boldsymbol{i}_{\widetilde{\boldsymbol{p}}}\right.$ and $\left.\boldsymbol{i}_{\widetilde{\boldsymbol{w}}}\right)$ represent the disturbing interactions caused by voltage and/or current harmonics (i.e., common or uncommon), as well as by asymmetries existing in either or both of these quantities. These oscillating portions do not contribute to the active power $(P)$ or to the reactive energy $(W)$. Hence, $\boldsymbol{i}_{\widetilde{\boldsymbol{p}}}$ e $\boldsymbol{i}_{\widetilde{\boldsymbol{w}}}$ are responsible for the instantaneous power oscillations in the electric circuit, as well as for additional losses.

From the load point of view, the decomposed portions shown in (10) and (11) do not represent any specific load behavior or characteristic; only on the condition of sinusoidal and balanced voltages, $\boldsymbol{i}_{\bar{p}}$ e $\boldsymbol{i}_{\overline{\boldsymbol{w}}}$ coincide with active and reactive balanced currents defined by $\mathrm{CPT}$. 
Since oscillations on reactive energy and instantaneous power can be caused by voltage and current distortions, as well as by voltage and current asymmetries, the reference signals for compensation can be represented by the sum of $\boldsymbol{i}_{\overline{\boldsymbol{w}}}, \boldsymbol{i}_{\widetilde{\boldsymbol{p}}}$ e $\boldsymbol{i}_{\widetilde{\boldsymbol{w}}}$ as presented in (12).

$$
\left[\begin{array}{l}
i_{a-A P F} \\
i_{b-A P F} \\
i_{c-A P F}
\end{array}\right]=\left[\begin{array}{l}
i_{a-\bar{w}} \\
i_{b-\bar{w}} \\
i_{c-\bar{w}}
\end{array}\right]+\left[\begin{array}{l}
i_{a-\tilde{p}} \\
i_{b-\tilde{p}} \\
i_{c-\tilde{p}}
\end{array}\right]+\left[\begin{array}{l}
i_{a-\widetilde{w}} \\
i_{b-\widetilde{w}} \\
i_{c-\widetilde{w}}
\end{array}\right] .
$$

It is noted in (12) that such references for compensation purposes can be applied in a selective manner, where the sum of its components $\boldsymbol{i}_{\widetilde{p}}$ e $\boldsymbol{i}_{\widetilde{w}}$ is related to the oscillations and $\boldsymbol{i}_{\bar{w}}$ is associated with the reactive energy (power) flow in the circuit. Hence, the components $\left(\boldsymbol{i}_{\widetilde{\boldsymbol{p}}}+\boldsymbol{i}_{\widetilde{\boldsymbol{w}}}\right)$ and $\left(\boldsymbol{i}_{\overline{\boldsymbol{w}}}\right)$ can be compensated independently by the MFGTI, or even by the combination of capacitor banks, which would compensate $\boldsymbol{i}_{\bar{w}}$, and the MFGTI ensuring compensation of $\boldsymbol{i}_{\widetilde{p}}+\boldsymbol{i}_{\widetilde{w}}$. Therefore, since the main goal of this study is to compensate the oscillations, the sum of the oscillating components will be taken as reference, in other words, $\boldsymbol{i}_{A P F}=\boldsymbol{i}_{\widetilde{p}}+\boldsymbol{i}_{\widetilde{\boldsymbol{w}}}$. Additionally, note that the above-mentioned novel current decomposition based on the CPT differs from [10], since it focuses on the perspective of operation seen by the power system (e.g., microgrid), intending to take advantage of MFGTIs to mitigate power oscillations.

\section{B. Generation of Reference Signals to Inject Active Power}

The major goal of a grid-tied inverter is to provide power conversion of the energy generated by a Local Energy Source (LES), injecting it into the grid. Consequently, active power injection should be supported, along with the proposed compensation of power oscillations. The generation of a current reference for the MFGTI, for the injection of active power, is based here on the synthesis of sinusoidal currents. According to this strategy, the injected current waveform should match the waveform of the positive sequence fundamental component of the PCC voltages $\left(\boldsymbol{v}_{m 1}^{+}\right)$. Thus, such strategy guarantees a smaller distortion level in the injected current, as explained in [10]. To achieve such behavior, the active current reference $i_{L E S}$ of the MFGTI is determined according to (13).

$$
\boldsymbol{i}_{L E S}=\frac{P_{L E S}}{\boldsymbol{V}_{1}^{2}}\left[\begin{array}{c}
v_{a 1}^{+} \\
v_{b 1}^{+} \\
v_{c 1}^{+}
\end{array}\right]=G_{L E S}\left[\begin{array}{c}
v_{a 1}^{+} \\
v_{b 1}^{+} \\
v_{c 1}^{+}
\end{array}\right]
$$

In (13), $\boldsymbol{V}_{\mathbf{1}}$ is the rms collective value of fundamental positive sequence voltages, $G_{L E S}$ is the equivalent conductance of the inverter, regarding active power injection, and $P_{L E S}$ is the liquid power generated from the LES, which is intended to be injected into the grid, being calculated by (14):

$$
P_{L E S}=\frac{1}{T} \int_{0}^{T} v_{D C}(t) i_{D C}(t) d t
$$

The fundamental voltage component at point of coupling of the MFGTI $\left(v_{m 1}\right)$ can be attained using a PLL, or through a band-pass filter with a narrow bandwidth tuned into the grid frequency. Considering that the grid frequency variation is relatively small, a band-pass filter was chosen to attain $v_{m 1}$, followed by the RMS $\left(V_{m 1}\right)$ value calculation. Consequently, as it will be later demonstrated, small variations in the grid frequency should not impair the operation of the MFGTI.

\section{Generation of Reference Signals for the Three-Phase Multi-functional Inverter}

Considering the reference signals shown in (12) and (13), the final current reference vector $\left(\boldsymbol{i}_{\text {ref }}^{*}\right)$, which is the term that is effectively synthesized by the MFGTI, is given by:

$$
\boldsymbol{i}_{r e f}^{*}=\boldsymbol{i}_{L E S}^{*}-\boldsymbol{i}_{A P F}^{*} .
$$

If the inverter was to operate only as a PEI, without doing the compensation of the instantaneous power oscillations, the signal $\boldsymbol{i}_{A P F}^{*}$ would be zero and the MFGTI would only inject the energy generated by the LES into the grid. On the other hand, as the component $\boldsymbol{i}_{\bar{w}}$ does not contribute to the instantaneous power oscillation, to completely compensate the instantaneous power oscillations, it is enough to assign the terms $\boldsymbol{i}_{\widetilde{p}}+\boldsymbol{i}_{\widetilde{\boldsymbol{w}}}$ to the compensation reference, as in (16).

$$
\boldsymbol{i}_{r e f}^{*}=\boldsymbol{i}_{L E S}^{*}-\boldsymbol{i}_{\tilde{p}}^{*}-\boldsymbol{i}_{\widetilde{w}}^{*}
$$

\section{CONTROL SYSTEM MODELING}

The control scheme for the MFGTI presented in this study is based on the modelling and approach proposed for threephase converters in [38]. The system presented in Figure 2 summarizes the topology of the adopted electrical circuit and configuration of the inverter. Also, the main electrical parameters are shown in Table I. Because the system presented in Figure 2 is a three-wire circuit with no neutral, the mesh control can be achieved considering the voltage and current values in only two phases of the three-phase system. For instance, for the converter, it is sufficient to control currents $i_{F a}$ and $i_{F b}$, since the current $i_{F c}$ is related to the previous currents, being $i_{F C}=-\left(i_{F a}+i_{F b}\right)$. Regarding the virtual point voltages, they can be obtained from the line voltage [33], according with (17). Thus, $v_{a b}$ and $v_{b c}$ could be measured and the third line voltage component is derived applying Kirchhoff's voltage law, having $v_{c a}=-\left(v_{a b}+\right.$ $\left.v_{b c}\right)$, for instance.

$$
\begin{aligned}
& v_{a}=\frac{1}{3}\left(v_{a b}+v_{a c}\right) \\
& v_{b}=\frac{1}{3}\left(v_{b a}+v_{b c}\right) \\
& v_{c}=\frac{1}{3}\left(v_{c b}+v_{c a}\right)
\end{aligned}
$$

The converter control system comprises two main control loops. The first is a fast loop used to control the converter output current, whereas the second is a voltage control loop that presents a slow dynamic response. The DC voltage control keeps the energy balance between the power delivered to the system at the converter's output side and the power at its DC bus [39]. Figure 3 shows the control strategy adopted with the control loops proposed for the MFGTI.

The current control is based on a proportional resonant controller tuned at certain harmonic frequencies [40], [41], as:

$$
G_{C}(s)=K_{C}+\sum_{h=1,3,5, \ldots, 11} \frac{2 K_{l_{P R}} \omega_{c_{P R}} s}{s^{2}+2 \omega_{c_{P R}} s+\left(h \omega_{o}\right)^{2}}
$$


In (18), $h$ is the harmonic order, $\omega_{o}$ is the grid fundamental frequency and $K_{C}, K_{I_{P R}}, \omega_{C_{P R}}$ are the proportional gain, integral gain and the resonant frequency band pass width, respectively. The $K_{I_{P R}}$ value is chosen to produce a high gain on harmonic frequencies and $\omega_{c_{P R}}$ should be designed to be small to improve the compensator selectivity.

The voltage controller used to maintain constant the DC bus voltage is a proportional-integral (PI) regulator, and it is given by (19):

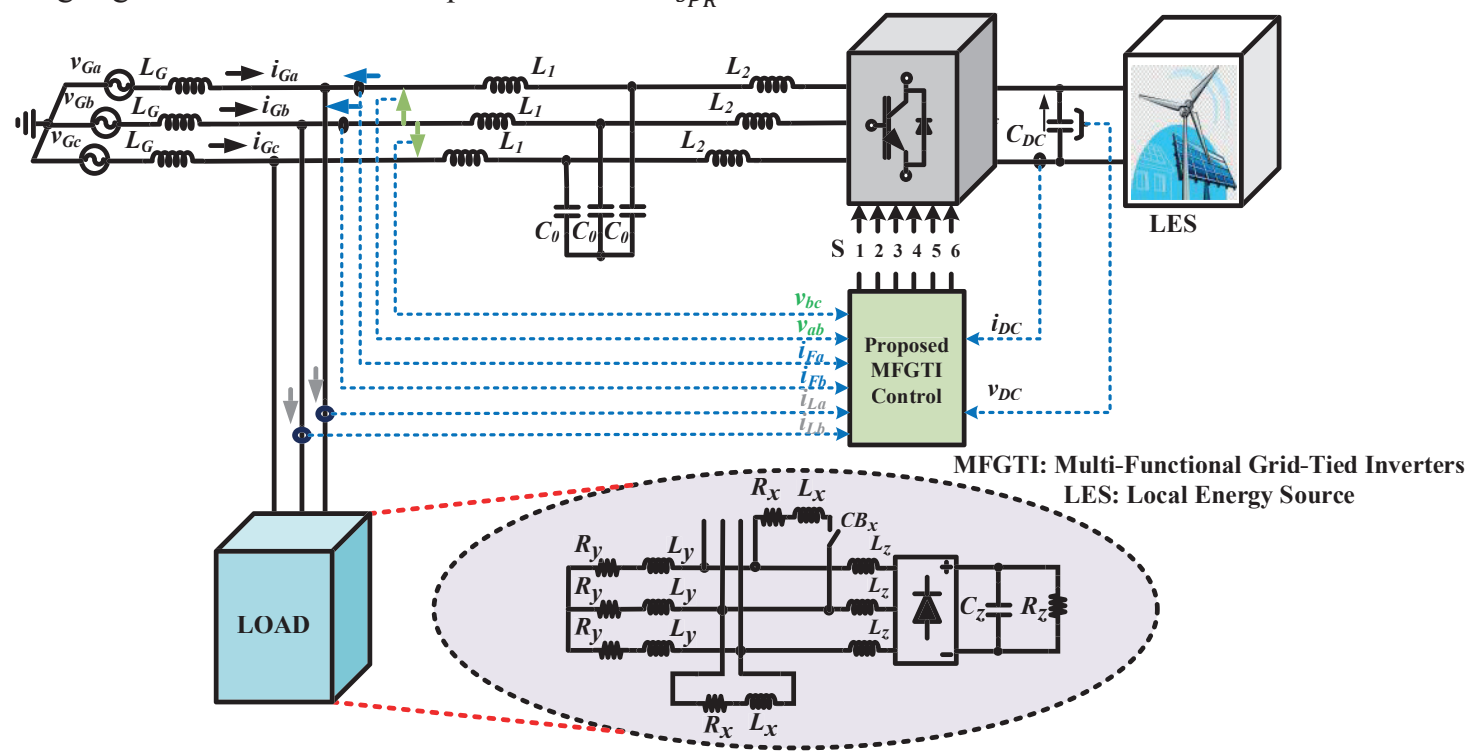

Fig. 2. Three-phase MFGTI connected to the electric grid.

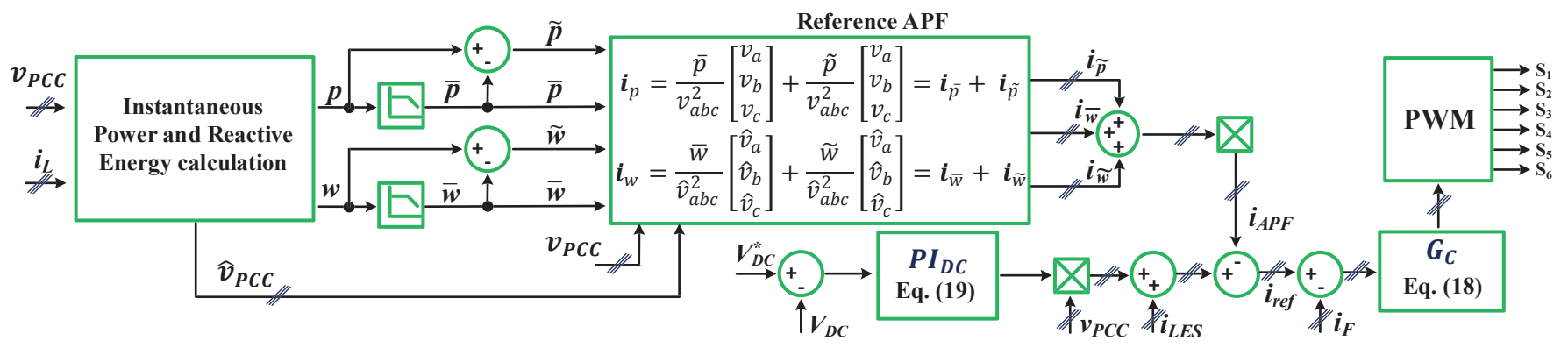

Fig. 3. Block diagram of the proposed multi-functional control structure.

TABLE I

Three-Phase Converter and System Parameters

\begin{tabular}{cccc}
\hline Parameter & Value & Parameter & Value \\
\hline Grid $\left(V_{\text {Line }}\right)$ & $220 \mathrm{~V} / 60 \mathrm{~Hz}$ & $L_{G} ; R_{G}$ & $0.25 \mathrm{mH} ; 0.1 \Omega$ \\
\hline$P_{\text {LES }}$ & $2.4 \mathrm{~kW}$ & $L_{1} ; R_{1}$ & $0.5 \mathrm{mH} ; 10 \mathrm{~m} \Omega$ \\
\hline$C_{0}$ & $3.3 \mu \mathrm{F}$ & $L_{2} ; R_{2}$ & $0.5 \mathrm{mH} ; 10 \mathrm{~m} \Omega$ \\
\hline$R_{x}, L_{x}$ & $5 \Omega ; 40 \mathrm{mH}$ & $L_{z}$ & $1 \mathrm{mH}$ \\
\hline$L_{y}, R_{y}$ & $70 \mathrm{mH} ; 30 \Omega$ & $R_{z} ; C_{z}$ & $50 \Omega ; 470 \mu \mathrm{F}$ \\
\hline
\end{tabular}

TABLE II

Controller Parameters

\begin{tabular}{cc}
\hline$K_{c}=2$ & $K_{l_{P R}}=100$ \\
\hline$\omega_{C_{P R}}=6.28[\mathrm{rad} / \mathrm{s}]$ & $\omega_{o}=377[\mathrm{rad} / \mathrm{s}]$ \\
\hline$K_{P_{D C}}=5$ & $K_{I_{D C}}=50$ \\
\hline & \\
$P I_{D C}(s)=K_{P_{D C}}+\frac{K_{I_{D C}}}{S}$
\end{tabular}

where $K_{\boldsymbol{P}_{\boldsymbol{D} C}}$ is the proportional controller gain, while $K_{\boldsymbol{I}_{\boldsymbol{D C}}}$ is the integral gain. The MFGTI was designed considering a switching frequency of $20 \mathrm{kHz}$. Table II shows the adopted values for the voltage and current controllers. The converter DC bus voltage is adjusted to $400 \mathrm{~V}$.

\section{SIMULATION RESULTS}

Aiming to assess the compensation strategy and the proposed control approach for the three-phase MFGTI, the circuit shown in Figure 2 was simulated using PSIM $^{\circledR}$. The implemented circuit comprises an electronic converter (DCAC) with an LCL output filter, a three-phase non-linear unbalanced load, and the electric grid.

The main goal expected for the simulation studies is to validate the feasibility of the proposed method in respect to providing operation under constant instantaneous power, and at the same time, consider active power injection into the grid. The dynamics of the system operation is described by several quantities (i.e., voltages, currents, power terms and instantaneous reactive energy), with respect to different measuring points: grid, load and MFGTI. Thus, the simulation results are presented for four different scenarios, as follows. 


\section{A. Scenario 1: MGTI only injecting active power}

In this first scenario, the goal is to operate the MFGTI only as a PEI (i.e., only providing injection of active power). From Figure 4, one can see that before $t<0.6 \mathrm{~s}$ the converter currents $\left(i_{F a}, i_{F b}, i_{F C}\right)$ are zero and the grid currents $\left(i_{G a}, i_{G b}, i_{G c}\right)$ are highly distorted and unbalanced. After turning on the PEI at $t>0.6 \mathrm{~s}$, the MFGTI currents become sinusoidal and synchronized with the PCC voltages. Hence, the mean grid component, $\bar{p}_{G}$ (i.e., active power), decreases while the grid currents continue to be distorted and unbalanced. Consequently, the oscillating components $\tilde{p}_{G}$ e $\widetilde{w}_{G}$ present a nonsinusoidal oscillation and continue to flow through the grid.
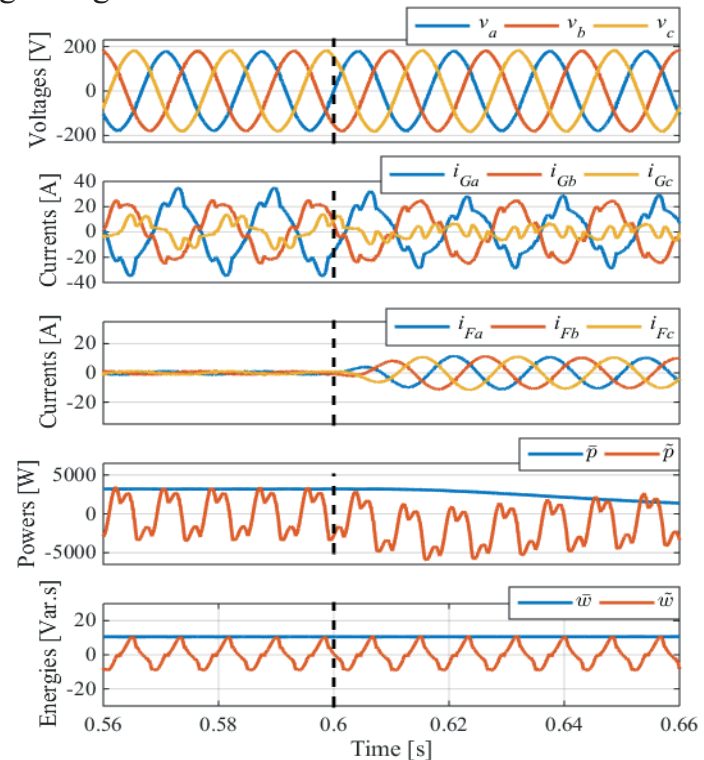

Fig. 4. Simulation results when the MFGTI is injecting only active power into the grid (Scenario 1). Top to bottom: PCC voltages and currents, MFGTI currents, PCC instantaneous active power, and instantaneous reactive energy.
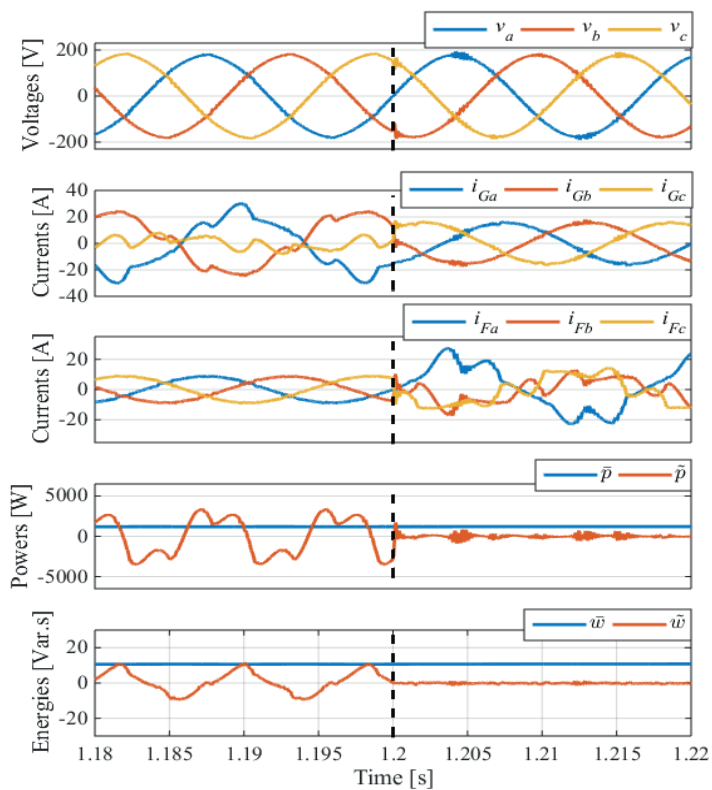

Fig. 5. Simulation results when the MFGTI is injecting active power and compensating the oscillations (Scenario 2). Top to bottom: PCC voltages and currents, MFGTI currents, PCC instantaneous active power and instantaneous reactive energy.
B. Scenario 2: MGTI injecting active power and compensating power oscillations

In this second scenario, the ancillary service capability (i.e., APF functionality) is added to the MFGTI, and the respective result can be seen in Figure 5. Before the APF function is activated $(t<1.2 \mathrm{~s})$ it can be observed that the harmonic distortion and current unbalance create a nonsinusoidal oscillation at the $\tilde{p}_{G}$ e $\widetilde{w}_{G}$ components. In addition, it can be seen a slightly distortion and voltage unbalance at the PCC, caused by the unbalanced non-linear load.
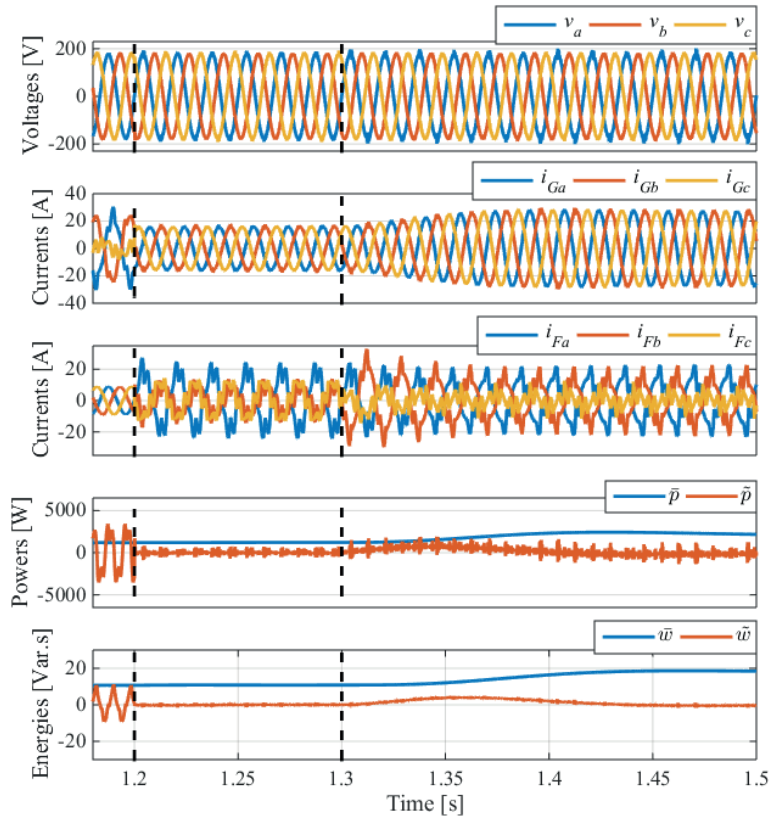

Fig. 6. Simulation results when the MFGTI is injecting active power and compensating the oscillations during a load step (Scenario 3). Top to bottom: PCC voltages and currents, MFGTI currents, PCC instantaneous active power, and instantaneous reactive energy.

After starting the APF service in the MFGTI at $t>1.2 \mathrm{~s}$, the current unbalances and harmonics generated by the nonlinear load (residual component) are compensated, which results in the elimination of all oscillating components $\left(\tilde{p}_{G}\right.$ and $\widetilde{w}_{G}$ ); therefore, improving the voltage waveforms at the PCC. The compensation results can also be observed at the grid current waveform that becomes sinusoidal and balanced. However, the grid current is still lagging the voltage because of the average reactive energy $\left(\bar{w}_{G}\right)$, which is not being compensated.

\section{Scenario 3: MFGTI operating under a load step}

In this case, a load step is simulated to demonstrate the dynamic behavior of the proposed strategy, being the results shown in Figure 6. Yet, the MFGTI operates injecting active power and providing compensation of oscillating power. As seen in Figure 2, an unbalanced load is connected to the grid at $1.3 \mathrm{~s}$ by switching on the circuit breaker $C B x$, causing the MFGTI to promptly adjust its current injection to maintain the compensation.

Note that, even though the average active power and reactive energy increased due to the connection of the unbalanced load, the instantaneous terms targeted on the compensation remained practically neglectable, as well as 
steady state operation was achieved after approximately 100 ms, which is basically the time constant from the DC controller (19). This important result indicates that the applied low pass filters for calculating $\bar{p}$ and $\bar{w}$ do not significantly impair on the system overall dynamic response.
D. Scenario 4: MGTI injecting active power and compensating power oscillations during frequency deviations

This last case is presented to demonstrate the operation of the MFGTI upon deviation in the grid frequency, which is likely to occur in scenarios such as the one of microgrids. The results are shown in Figure 7. This case is a continuation of the result shown in Scenario 2, considering the same parameters

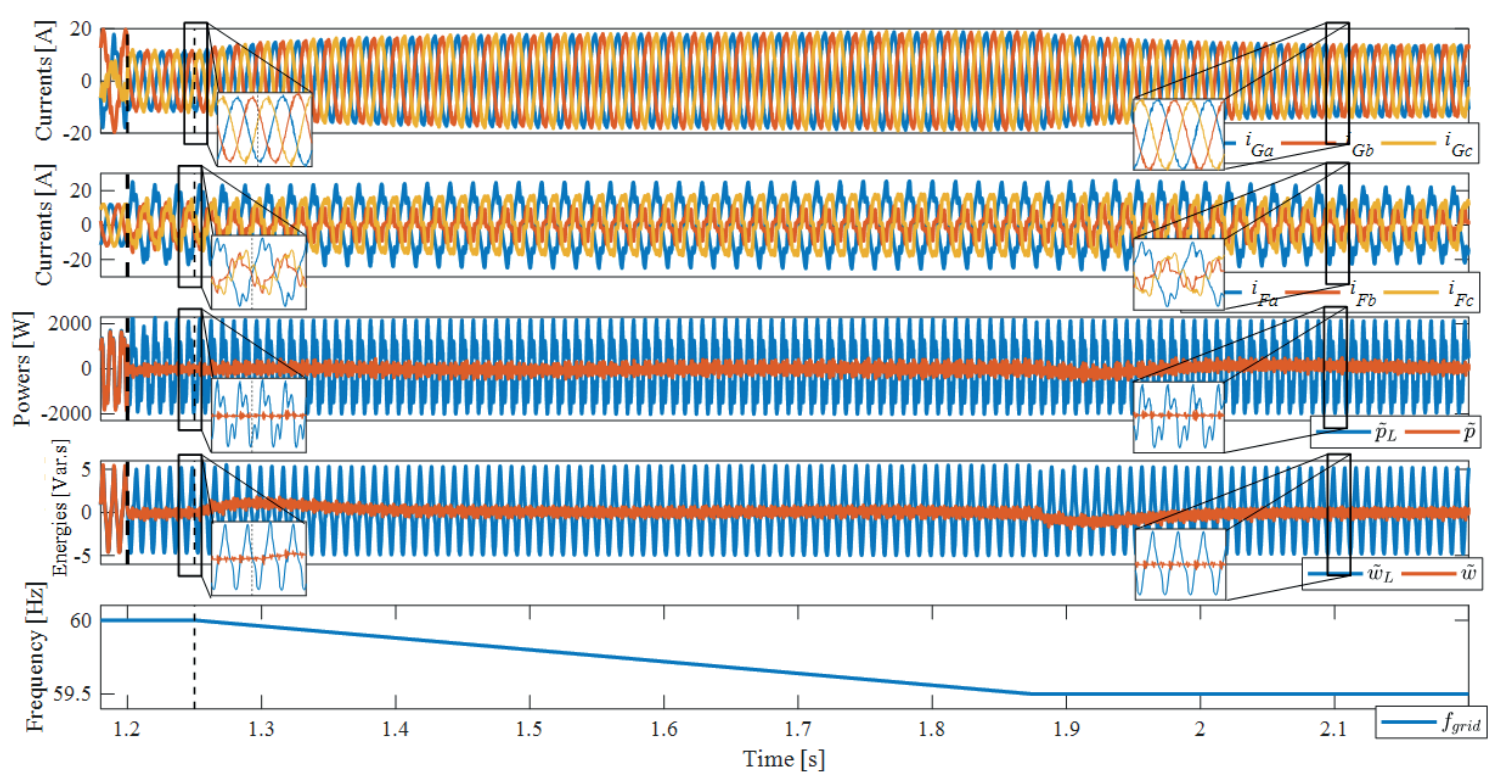

Fig. 7. Simulation results with grid frequency deviation when the MFGTI is injecting active power and compensating the oscillations (Scenario 4). Top to bottom: PCC currents, MFGTI currents, load and PCC instantaneous oscillating active power, load and PCC instantaneous oscillating reactive energy, and grid frequency.

of Figure 5, on which the MFGTI operates injecting active power and compensating for the power oscillations.

As in Figure 5, when $t>1.2 \mathrm{~s}$, the MFGTI starts its operation and the active and reactive power oscillations are compensated (i.e., see the load and PCC active and reactive instantaneous powers in Figure 7), resulting in sinusoidal currents at the PCC. Nonetheless, when $t>1.25 \mathrm{~s}$, the grid frequency starts to deviate. Herein, a variation up to the lower boundary of frequency deviation within [42] (i.e., 59.5 $\mathrm{Hz}$ ) was emulated as example.

As the grid frequency transits from $60 \mathrm{~Hz}$, the amplitude of the PCC current slightly increases due to the constant active power generation by the MFGTI. On the other hand, and most importantly to the focus of this paper, note that the MFGTI rides through the frequency variation without losing its capability to perform compensation of the active and reactive oscillating powers. The zoom-in-view of the steadystate operation of the system during $59.5 \mathrm{~Hz}$ shows that, similar to the previous instants, the PCC current still resembles a sinusoidal waveform, and the oscillations in the powers are practically neglectable. Additionally, overcurrents were not present as a result of such operation.

\section{EXPERIMENTAL RESULTS}

Aiming at validating the proposed control strategy of a three-phase MFGTI compensating for oscillating instantaneous power, a laboratory prototype shown in Figure
8 was developed. This setup comprises a $3.6 \mathrm{kVA}$ inverter, presenting LCL output filter. The control algorithms were implemented on a TMS320F28335 floating-point digital signal controller (DSC). The MFGTI's and grid's parameters are presented in Table III. A non-linear load is connected at PCC following the same disposition of Figure 1. Such load is composed of a three-phase diode bridge rectifier feeding a resistor (i.e., $R_{L}=62 \Omega$ ) at its DC side. An AC smoothing inductor $\left(L_{L}=1 \mathrm{mH}\right)$ is connected at the input of diode bridge. The DC bus of the MFGTI is fed by a constant voltage source. Yet, the $\mathrm{AC}$ grid is emulated by a controllable power supply. Figure 8 shows the main elements of the prototype build.

TABLE III

MFGTI's and Grid's Parameters

\begin{tabular}{cc}
\hline Parameters & Value \\
\hline Switching frequency $\left(f_{S W}\right)$ & $20 \mathrm{kHz}$ \\
\hline Sampling frequency $\left(f_{S}\right)$ & $20 \mathrm{kHz}$ \\
\hline Fundamental frequency $(f)$ & $60 \mathrm{~Hz}$ \\
\hline Voltage grid (line voltage) $\left(V_{\text {grid }}\right)$ & $110 \mathrm{~V}$ \\
\hline$L_{\text {grid }}$ & $1 \mathrm{mH}$ \\
\hline$L_{I E P}$ & $1 \mathrm{mH}$ \\
\hline DC bus voltage $\left(V_{D C}\right)$ & $400 \mathrm{~V}_{C C}$ \\
\hline
\end{tabular}




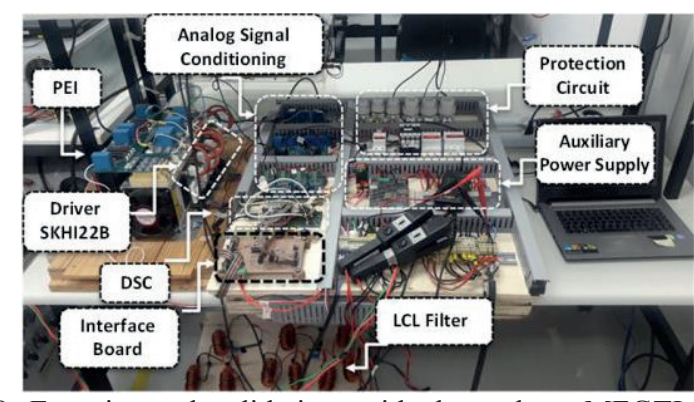

Fig. 8. Experimental validation, with three-phase MFGTI, with a DSP based implementation.

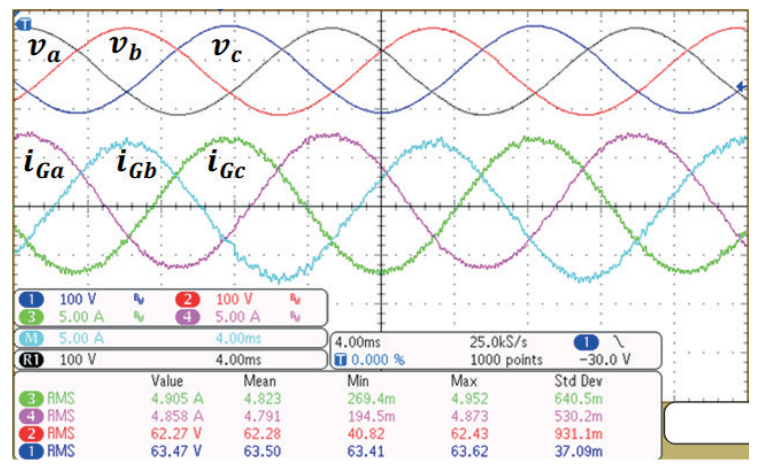

Fig. 9. Voltages and currents at the PCC when the MFGTI is connected to the grid injecting active power.

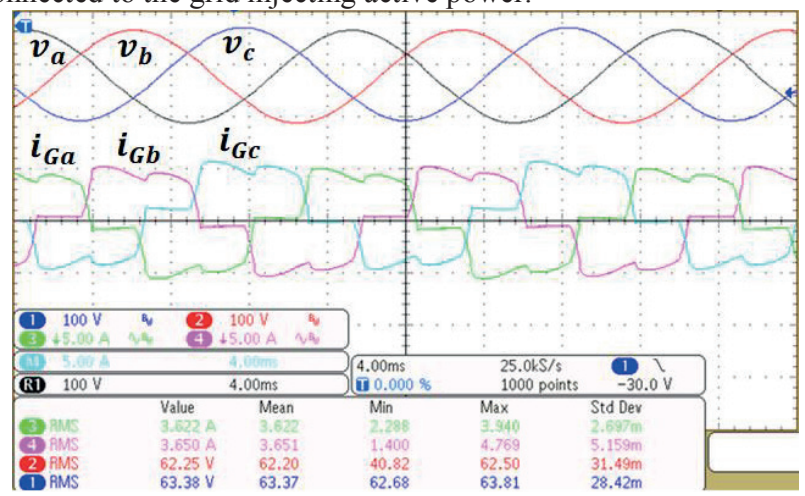

Fig. 10. Voltages and currents at the PCC when only the non-linear is connected.

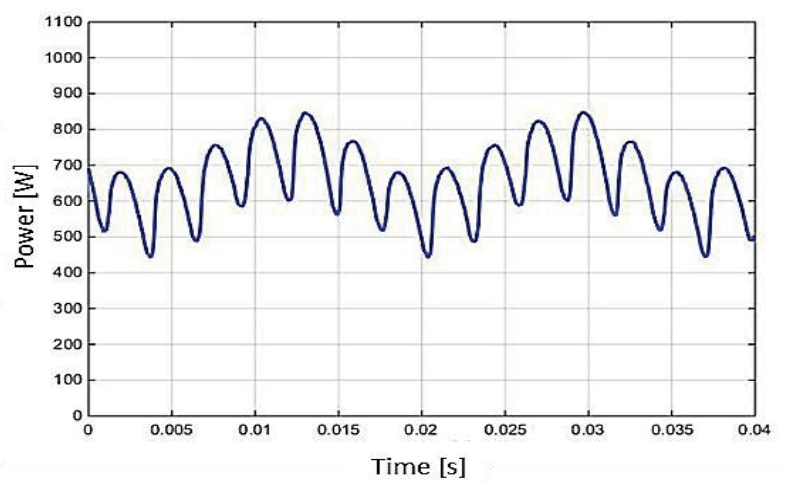

Fig. 11. Instantaneous oscillating power saw at the PCC when only the load is connected to the grid.

Firstly, Figure 9 shows waveforms of PCC voltage and MFGTI current when it is only acting as a PEI, injecting 1 $\mathrm{kW}$

into the grid. Note that the injected currents in-phase concerning the PCC voltages present low distortion.
Figure 10 shows voltages and currents seen at the PCC, considering that the MFGTI is not connected to PCC; consequently, neither compensation functionalities nor active current injection occurs. Note that the grid voltages are sinusoidal, and the currents drawn by the non-linear load are highly distorted. The instantaneous power (calculated using the data points of Figure 10) seen at the PCC is presented in Figure 11. It is understood that $p(t)$ has a nonsinusoidal oscillation as a consequence of the harmonic currents drawn by the load.

Finally, Figures 12 and 13 show the waveforms produced when the MFGTI is connected to the PCC acting as PEI (injecting active power) and performing compensation of oscillating instantaneous power. Note that MFGTI's currents waveforms, shown in Figure 12, are nonsinusoidal since the MFGTI is injecting active power, as previously shown in Figure 11, along with the compensation of CPT's terms $\widetilde{\boldsymbol{p}}, \widetilde{\boldsymbol{w}}$ and $\overline{\boldsymbol{w}}$ (i.e., compensation of oscillating instantaneous power and reactive energy.).

The resulting currents at the grid side can be seen in Figure 13 as well as PCC voltages. Note that, compared to Figure 9, the PCC currents became less distorted. By looking into the oscillating instantaneous power in Figure 14, one can note the reduction of the low-frequency oscillations previously existing in Figure 10. This indicates that the oscillating instantaneous power was reduced due to the action of the MFGTI.

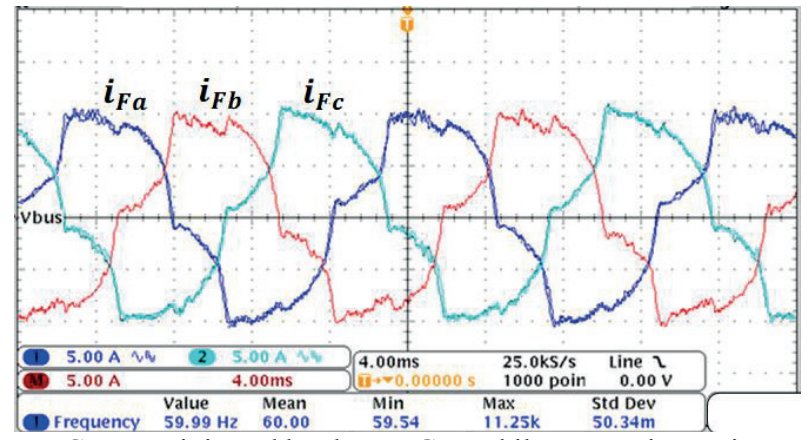

Fig. 12. Currents injected by the MFGTI while processing active power and compensating for oscillating instantaneous power.

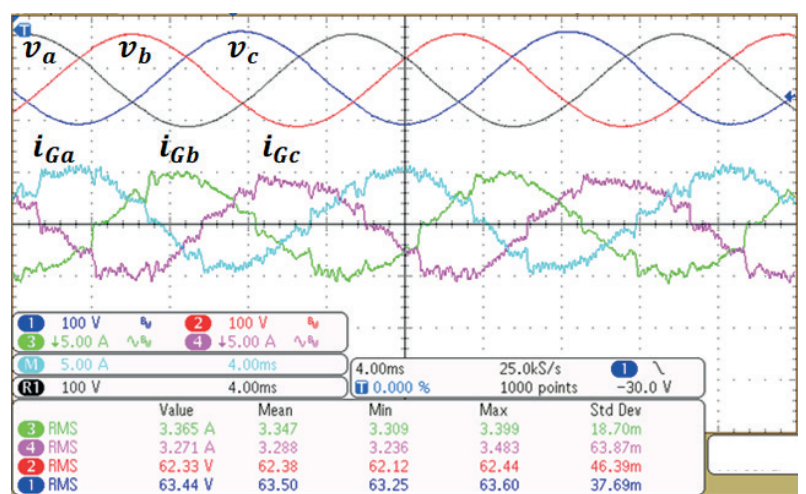

Fig. 13. Voltages and currents at the PCC when the MFGTI is injecting active power and compensating for oscillating instantaneous power. 


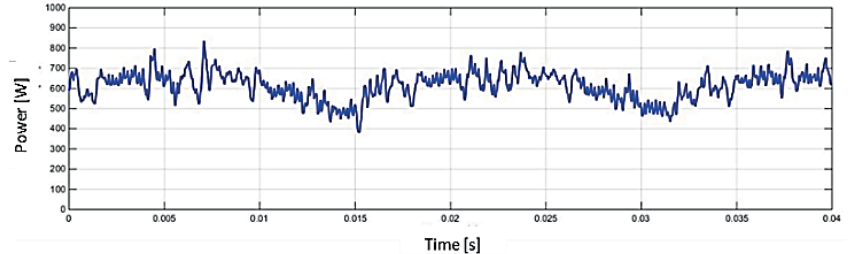

Fig. 14. Instantaneous oscillating power at the PCC when the MFGTI is injecting active power and providing compensation.

\section{CONCLUSIONS}

In this paper, the authors presented a novel strategy of using grid-connected multi-functional power electronic converters for injecting active power into the grid and compensating instantaneous power oscillations. The proposed control is based on instantaneous and average terms $(p(t), w(t), \bar{p}(t)$ and $\bar{w}(t))$ of the Conservative Power Theory (CPT).

As shown in the simulation results, the proposed control strategy allows not only the injection of active power, but also supports power system operation at constant instantaneous power, $p(t)=\bar{p}(t)$, even under different linear/non-linear unbalanced load configurations. This feature is critical since the instantaneous power oscillations are undesirable in any power system due to rotating electric machines deterioration, and operation issues regarding power quality, especially on scenarios of weak electrical grids such as microgrids. Experimental results also demonstrated that the control strategy could be embedded onto digital processors, being practically implemented for purposes of compensation instantaneous oscillating power.

The proposed control strategy can be understood as an alternative to other traditional techniques that provide compensation for oscillating power components in electrical systems. For instance, it is noticeable that the power terms decomposed by this approach resemble the ones defined within the PQ theory. Nonetheless, the CPT provides such decomposition directly on the abc frame, without requiring axis transformations. It is also worth mentioning that the approach does not require the implementation of synchronization algorithms, as needed by other methods based on $\mathrm{dq}$ transformations, consequently eliminating particular stability concerns. On the other hand, the application of low-pass filters is inherent to the proposed approach, as in most strategies found in literature, which may cause slow accommodation times for the compensation behavior if not properly designed.

Finally, considering the achieved compensation results, future work is needed to verify the feasibility of the proposed strategy under distorted and unbalanced conditions, not only at PCC but also at the voltage source (grid-side). Thus, power system operation at constant instantaneous power is expected even under adverse conditions. Yet, extensions of this work intend to further evaluate the singularities of the proposed methodology, exploring its advantages and disadvantages when compared to other methods in the literature.

\section{ACKNOWLEDGEMENTS}

This work was supported by the São Paulo Research Foundation (FAPESP) (Grants, 2017/22629-9, 2017/209875, 2017/24652-8, 2016/08645-9), by the National Council for Scientific and Technological Development (CNPq) (Grants $311332 / 2018-8,313806 / 2019-5$ and 424853/2016-7). This study was financed in part by the Coordenação de Aperfeiçoamento de Pessoal de Nível Superior - Brasil (CAPES) - Finance Code 001.

\section{REFERENCES}

[1] A. U. Krismanto, M. Nadarajah and O. Krause, "Influence of renewable energy based microgrid on low frequency oscillation of power systems," 2015 IEEE PES Asia-Pacific Power and Energy Engineering Conference (APPEEC), Brisbane, QLD, pp. 1-5, 2015.

[2] D. Castro, T. Soares and M. Matos, "Stochastic Energy and Reserve Market in a Microgrid Environment," 2019 IEEE Milan PowerTech, Milan, Italy, pp. 1-6, 2019.

[3] M. Di Somma, G. Graditi and P. Siano, "Optimal Bidding Strategy for a DER Aggregator in the DayAhead Market in the Presence of Demand Flexibility," in IEEE Trans. Ind. Electron., vol. 66, no. 2, pp. 15091519, Feb. 2019.

[4] T. Strasser et al., "A Review of Architectures and Concepts for Intelligence in Future Electric Energy Systems," in IEEE Trans. Ind. Electron., vol. 62, no. 4, pp. 2424-2438, April 2015.

[5] Xue et al., "On a Future for Smart Inverters with Integrated System Functions," $9^{\text {th }}$ IEEE Int. Sym. Power Electron. Dist. Gen. Syst., Charlotte, pp. 1-8, 2018.

[6] T. Samad and A. M. Annaswamy, "Controls for Smart Grids: Architectures and Applications," Proceedings of the IEEE, vol. 105, no. 11, pp. 2244-2261, Nov. 2017.

[7] Z. Zeng, X. Li and W. Shao, "Multi-functional gridconnected inverter: upgrading distributed generator with ancillary services," IET Renew. Power Gen., vol. 12, no. 7, pp. 797-805, May 2018.

[8] W. Choi, W. Lee, D. Han and B. Sarlioglu, "New Configuration of Multifunctional Grid-Connected Inverter to Improve Both Current-Based and VoltageBased Power Quality," IEEE Trans. Ind. Appl., vol. 54, no. 6, pp. 6374-6382, Nov.-Dec. 2018.

[9] Y. Yang, F. Blaabjerg, H. Wang and M. G. Simões, "Power control flexibilities for grid-connected multifunctional photovoltaic inverters," IET Renew. Power Gen., vol. 10, no. 4, pp. 504-513, April 2016.

[10] F. P. Marafão, D. I. Brandão, A. Costabeber and H. K. M. Paredes, "Multi-task control strategy for grid-tied inverters based on conservative power theory," IET Renew. Power Gen., vol. 9, no. 2, pp. 154-165, 2015.

[11] R. Bhoyar and S. Bharatkar, "Potential of MicroSources, Renewable Energy sources and Application of Microgrids in Rural areas of Maharashtra State India," Energy Procedia, vol. 14, pp. 2012-2018, 2012.

[12] X. Zhao-xia, Z. Mingke, H. Yu, J. M. Guerrero and J. C. Vasquez, "Coordinated Primary and Secondary 
Frequency Support Between Microgrid and Weak Grid," IEEE Trans. Sust. Energy, vol. 10, no. 4, Oct. 2019.

[13] D. E. Olivares et al, "Trends in Microgrid Control," IEEE Trans. Smart Grid, vol. 5, pp. 1905-1919, July 2014.

[14] D. I. Brandao, P. Tenti, T. Caldognetto, and S. Buso, "Control of Utility Interfaces in Low-voltage Microgrids," Brazilian J. Power Electron. - SOBRAEP, vol. 20, no. 4, pp. 373-382, Nov. 2015.

[15] R. Palma-Behnke et al, "A Microgrid Energy Management System Based on the Rolling Horizon Strategy," IEEE Trans. Smart Grid, vol. 4, no. 2, pp. 996-1006, 2013.

[16] J. M. Carrasco et al, "Power Electronic Systems for the Grid Integration of Renewable Energy Sources: A Survey," IEEE Trans. Ind. Electron., vol. 53, pp. 1002 1016, June 2006.

[17] M. Amin, Q. Zhong, "Resynchronization of Distributed Generation Based on the Universal Droop Controller for Seamless Transfer Between Operation Modes," IEEE Trans. Ind. Electron., vol. 67, pp. 7574-7582, Sep. 2020.

[18] G.-H. Kim et al, "A Novel Three-phase Four-leg Inverter Based Load Unbalance Compensator for Stand-alone Microgrid," Elec. Power and Energy Syst., vol. 65, p. 70-75, Feb. 2015.

[19] F. Z. Peng et al, "Harmonic and Reactive Power Compensation Based on the Generalized Instantaneous Reactive Power Theory for Three-Phase Four-Wire Systems," IEEE Trans. Power Electron., vol. 13, Nov. 1998.

[20] H. Akagi, Y. Kanazawa, A. Nabae, "Instantaneous Reactive Power Compensator Comprising Switching Devices Without Energy Storage Components," IEEE Trans. Ind. Appl., vol. IA-20, pp. 625-630, May 1984.

[21] E. H. Watanabe et al, "Instantaneous p q Power Theory for Control of Compensators in Micro-grids," in Proc. 2010 International School on Nonsinusoidal Currents and Compensation, Lagow, pp. 17-26, 2010.

[22] Y. Sun et al, "Microgrid Tie-line Power Fluctuation Mitigation with Virtual Energy Storage," The Journal of Engineering, vol. 2019, pp. 1001-1004, April 2019.

[23] Z. Miao, L. Fan, D. Osborn, S. Yuvarajan, "Control of DFIG-Based Wind Generation to Improve Interarea Oscillation Damping," IEEE Trans. Energy Conv., vol. 24, pp. 415-422, June 2009.

[24] L. Fan, Z. Miao, "An Explanation of Oscillations Due to Wind Power Plants Weak Grid Interconnection," IEEE Trans. Sust. Energy, vol. 9, pp. 488-490, January 2018.

[25] D. Gautam, V. Vittal, R. Ayyanar, T. Harbour, "Supplementary Control for Damping Power Oscillations Due to Increased Penetration of Doubly fed Induction Generators in Large Power Systems", in Proc. IEEE/PES Power Sys. Conf. Exp., PSCE 2011.

[26] J. P. Bonaldo, H. K. M. Paredes, J. A. Pomilio, "Control of Single-Phase Power Converters Connected to LowVoltage Distorted Power Systems with Variable
Compensation Objectives", IEEE Trans. Power Electron., vol. 31, pp. 2039-2052, March 2016.

[27] J. He, Y. W. Li, F. Blaabjerg, X. Wang, "Active Harmonic Filtering Using Current-controlled, Gridconnected DG Units with Closed-loop Power Control," IEEE Trans. Power Electron., vol. 29, pp. 642-653, February 2014

[28] J. He, Y. W. Li, M. S. Munir, "A Flexible Harmonic Control Approach Through Voltage Controlled DG-grid Interfacing Converters," IEEE Trans. Ind. Electron., vol. 59, pp. 444-455, January 2012.

[29] F. Z. Peng, "Harmonic Sources and Filtering Approaches," IEEE Ind. Appl. Mag., vol. 7, pp. 18-25, July 2001.

[30] P. Tenti, H. K. Morales-Paredes, P. Mattavelli, "Conservative Power Theory, a Framework to Approach Control and Accountability Issues in Smart Microgrids," IEEE Trans. Power Electron., vol. 26, pp. 664-673, March 2011.

[31] D. Dong et all, "Analysis of Phase-Locked Loop LowFrequency Stability in Three-Phase Grid-Connected Power Converters Considering Impedance Interactions," IEEE Trans. Ind. Electron., vol. 62, no 1, pp. 310-321, Jan. 2015

[32] H. K. Morales-Paredes, J. A. Olímpio Filho, A. M. S. Alonso, J. P. Bonaldo, F. P. Marafão, M. G. Simões. 3Phase Multi-Functional Grid-Tied Inverter for Compensation of Oscillating Instantaneous Power. In: 15th Brazilian Power Electron. Conf. and 5th IEEE South. Power Electron. Conf., 2019, Santos. Proceedings of COBEP/SPEC 2019, p. 1-6, 2019.

[33] P. Tenti, P. Mattavelli and H. K. Morales, "Conservative Power Theory, Sequence Components and Accountability in Smart Grids," Prz. Elektrotech, vol. 6, pp. 30-37, 2010.

[34] F. P. Marafão, D. I. Brandão, F. A. S. Gonçalves, and H. K. Morales Paredes, "Decoupled Reference Generator for Shunt Active Filters Using the Conservative Power Theory," J. Control Autom. Electr. Syst, pp. 522-534, August 2013.

[35] D. I. Brandão, H. K. Morales-Paredes, A. Costabeber, F. P. Marafão, "Flexible Active Compensation Based on Load Conformity Factors Applied to Nonsinusoidal and Asymmetrical Voltage Conditions", IET Power Electron., vol. pp. 1-9, February 2015.

[36] H. Akagi, E. H. Watanabe and M. Aredes, Instantaneous Power Theory and Applications to Power Conditioning, New Jersey, 2007.

[37] A. V. Oppenheimer and R. W. Schafer, "Discrete-Time signal processing”. PrenticeHall International Inc. 1989.

[38] Yi Tang, P.C. Loh, Peng Wang, F.H. Choo, F. Gao. "Generalized Design of High Performance Shunt Active Power Filter with Output LCL Filter", IEEE Trans. Ind. Electron., vol.59, March 2012.

[39] M. G. Villalva, J. R. Gazoli, E. R. Filho, "Modeling and Control of a Three-phase Isolated Grid-connected Converter fed by a Photovoltaic Array," in Proc. Brazilian Power Electron. Conf., Dec. 2009. 
[40] S. Buso, P. Mattavelli, "Digital Control in Power Electronics", First edition, Morgan \& Claypoo, United States of America, 2006.

[41] R. Teodorescu, F. Blaabjerg, M. Liserre, P. C. Loh, "Proportional-Resonant Controllers and Filters for Grid-connected Voltage-source Converters," Electric Power Appl., IEE Proceedings, vol. 153, no.5, pp.750762, September 2006.

[42] ANEEL, "Procedimentos de Distribuição de Energia Elétrica no Sistema Elétrico Nacional - PRODIST: Módulo 8 - Qualidade de Energia”, Jan. 2018.

\section{BIOGRAPHIES}

José de Arimatéia Olímpio Filho, received the B.S. degree in Electrical Engineering from the Federal University of Mato Grosso do Sul, Brazil, in 2017. He received the M.S. degree in Electrical Engineering from the São Paulo State University (UNESP/FEB), Brazil, in 2019. His main interests are power electronics and power quality.

Helmo Kelis Morales Paredes, received the B.S. degree from the San Agustin National University Arequipa, Peru, in 2002, and the M.Sc. and Ph.D. degrees from UNICAMP, Brazil, in 2006 and 2011, respectively, all in electrical engineering. In 2009, he joined the Power Electronics Group, University of Padova, Italy, as a Visiting Student. In 2014, he joined the PEMC Group, University of Nottingham, U.K., as a Visiting Scholar. In 2018, he joined the Colorado School of Mines, USA, as a Visiting Scholar. Since 2011, he has been with UNESP, as an Associated Professor and he is the Leader of the Group of Automation and Integrating Systems. His current research interests include power theories under nonsinusoidal condition, harmonics propagation, power quality, accountability, revenue metering, and power electronics applied for distributed generation and renewable energy systems.

Dr. Morales-Paredes was a recipient of a Prize Paper Award from the IEEE TRANSACTIONS ON POWER ELECTRONICS in 2011 and currently he is an Associate Editor for the IEEE Latin America Transactions. He is a member of SOBRAEP, SBA and IEEE.

Augusto Matheus dos Santos Alonso, received the B.S. degree in automation and control engineering from the Federal University of Ouro Preto, in 2014, with a sandwich period at the University of New Mexico, USA, between 2012-2013. He received the M.S. degree in electrical engineering from the São Paulo State University (UNESP), Brazil, in 2018. Currently, he is a FAPESP scholar working toward a double Ph.D. degree at UNESP and at the
Norwegian University of Science and Technology, Norway. In 2019, he was a recipient of the SOBRAEP award for the best M.S. thesis of the year. His main interests are control of power converters, microgrid control, power quality, and energy policies.

Jakson Paulo Bonaldo, received the B.S. degree in electrical engineering from Federal University of Mato Grosso, Cuiaba, in 2008, and M.S. and Ph.D. degrees in electrical engineering from the University of Campinas, Campinas, Brazil, in 2010 and 2015, respectively. From 2010 to 2011 he was with Padtec Optical Components and Systems as a firmware engineer. From 2013 to 2018 he was with the Department of Electronics at Federal University of Technology - Parana. Since 2018 he is with the Department of Electrical Engineering at Federal University of Mato Grosso as assistant professor. His main interests are power quality, smart microgrid and renewable energy. $\mathrm{He}$ is member of SOBRAEP.

Fernando Pinhabel Marafão, received the B.S. degree in electrical engineering from UNESP, Brazil, in 1998, and the M.Sc. and Ph.D. degrees from UNICAMP, Brazil, in 2000 and 2004, respectively. In 2002, he joined the Power Electronics Group, University of Padova, Italy, as a visiting student. In 2013, he joined the Colorado School of Mines, USA, as a Visiting Scholar. Since 2005, he has been with UNESP, as an Associate Professor with the Group of Automation and Integrating Systems. His current research interests include smart grid technologies, distributed generation, energy management, power electronics and power theories. He is a member of SOBRAEP, SBA and IEEE.

Marcelo Godoy Simões, received the B.Sc. and M.Sc. degrees from USP, Brazil, the Ph.D. degree from The University of Tennessee, USA, in 1985, 1990 and 1995, respectively. He was a US Fulbright Fellow for AY 2014-15, Aalborg University, Denmark. He is a pioneer to apply neural networks and fuzzy logic in power electronics, motor drives, and renewable energy systems. His leadership in modeling fuel cells is internationally and highly influential in providing a basis for further developments in fuel cell automation control in many engineering applications.

Dr. Simões was a recipient of the "2018 IET Renewable Power Generation Premium Award." He is an IEEE Fellow, Class of 2016, with the citation: "for applications of artificial intelligence in control of power electronics systems." $\mathrm{He}$ is currently serving as the Chair for the IEEE Industrial Electronics Society Denver Chapter. 